\title{
Qualitative Inquiry of Adolescents' Experience of Ethical Issues During Enrollment and Adherence to Antiretroviral Therapy (ART) in Temeke Regional Hospital, Tanzania
}

Renatha Sillo Joseph ( $\sim$ renatha1972@yahoo.com )

Muhimbili University of Health and Allied Sciences

Gladys Reuben Mahiti

Muhimbili University of Health and Allied Sciences

Gasto Frumence

Muhimbili University of Health and Allied Sciences

Connie M. Ulrich

University of Pennsylvania

\section{Research Article}

Keywords: Adolescents, ethical issues, HIV care and treatment, adherence to Antiretroviral Therapy.

Posted Date: July 30th, 2021

DOI: https://doi.org/10.21203/rs.3.rs-737200/v1

License: (a) (i) This work is licensed under a Creative Commons Attribution 4.0 International License. Read Full License 


\section{Abstract \\ Background}

Adolescents living with HIV experience ethical challenges including lack of involvement in their care as well nondisclosure of HIV status which leads to poor adherence to antiretroviral therapy (ART). Parents have the authority over their children but during adolescence there is increase sense of independence. Whose decision should be followed in case of disagreement. Aim of the study is to explore experience of ethical issues identified by adolescents age 10 to 19 years attending HIV care and treatment in Temeke regional referral hospital.

\section{Methods}

Exploratory descriptive qualitative design was employed at Temeke regional referral hospital in Care and Treatment Centre in the Out-Patient Department with adolescents living with HIV 10 to19 years of age. A total of 22 adolescents participated in in-depth face-to-face interviews after obtaining parental consent and adolescent assent. All interviews were audio-taped, transcribed verbatim in Swahili, and back translated to English. Data analysis included both inductive and deductive thematic analysis and rigor was maintained using standards of qualitative research.

\section{Results}

Qualitative themes identified included: lack of participation in decisions about HIV care and treatment; issues around disclosure of HIV status, such as delays in disclosure to the adolescent and disclosure to other persons and benefits and harms of such disclosures; and factors supporting and interfering with adherence to ART, such as parental support, organizational (clinic) support and problems, and self-stigmatization and shame.

\section{Conclusion}

Lack of adolescents' involvement in their care decision making and delayed disclosure are identified ethical concerns leading to poor adolescents' adherence to ART. Early disclosure to adolescents is beneficial the same as disclosure to others especially teachers helped adolescents at school to take their medication properly. Disclosure to others apart from adolescents lead to stigma and discrimination for some adolescents.

\section{Background}

Adolescence is the developmental period when capacity for decision making evolves (1). Indeed, adolescents have an increasing need for their involvement in making decisions related to their care and well-being $(2,3)$ Health care providers and parents should foster adolescents' autonomy and decision-making capacity that supports their ability to make informed judgments (4). Adolescent involvement in their own health care decision making can create a positive relationship between the doctor, the patient, and the parent, enhancing adolescent's satisfaction with their care and trust between all parties (5). Even though adolescents should participate in decisions that affect their health and well-being, parents have the authority to make decisions for their sons and 
daughters (UNICEF-Tanzania, 2011). However, adolescents make up 24\% of all Tanzanians (7), and the prevalence of human immunodeficiency syndrome (HIV) infection in adolescents was $6 \%$ in 2013 (8). It is predicted that the adolescent population will continue to expand and to be disproportionately impacted by HIV and the acquired immunodeficiency syndrome (AIDS) epidemic, with only $35.4 \%$ of adolescent females and $15 \%$ of adolescent males being HIV tested in 2015 (9).

When an adolescent is ill, parents, the adolescent, and health care providers may disagree about what should be done or whose decision should be followed (10). Adolescents' growing sense of independence may lead to conflict between them and their parents or designated care givers (11). This is the case, for example, when an adolescent has a diagnosis of HIV and/or AIDS ethical issues often arise in African setting (Knapp van Bogaert \& Ogunbanjo, 2011). Adolescence is a time when decisions may need to be made about HIV testing and/or disclosure of previously known HIV status to the adolescent. In Tanzania in 2019, 11\% of children were born with HIV infection via perinatal transmission (12). Many parents who are aware of their infant's HIV infection choose not to tell the young child, and instead administer daily medications throughout childhood. However, by adolescence, there are ethical issues regarding continued nondisclosure of HIV status to the affected individual. Such disclosure is often surrounded by parent's fear of emotionally distressing a child (13). Adolescence is also a time when individuals enroll in HIV care and treatment programs and learn about the importance of adherence to care. And adolescence is a time when HIV stigma and discrimination may particularly affect the developing adolescent and their families (14).

Ensuring adherence to antiretroviral therapy (ART) among adolescents can present challenges. A qualitative study conducted in Canada with 34 HIV-positive young people documented their confusion and misunderstanding of HIV treatment; some felt they had no choice about treatment, some cited disruptions to their lives, and some reported difficulties in adhering to ART (15). Many factors may influence adolescents' adherence to ART, including their motivations to receive care, the serious nature of their illness, communication styles in the clinical interaction, peer support, and structural issues (16). Among factors influencing adherence, knowing one's HIV status improves adolescents' adherence to ART (17). However, as noted, some adolescents infected perinatally may not know their HIV status; when there is no proper disclosure and sex education, these adolescents may infect others (18). In fact, a qualitative study at Mbagala tertiary health facility in Tanzania found low rates of HIV status disclosure to children and adolescents under age 15 (19).

Risky behavior and curiosity expose adolescents to the potential for unprotected sex and increased risk of HIV infection (20). In Tanzania, adolescents' enrollment in care and treatment for HIV and AIDS is low (21). On top of the low enrollment, there is also a high rate of loss to follow-up and poor adherence to medication, which further increase HIV infection incidences and mortality. Thus, there is a great need to understand the ethical issues that adolescents face because of their HIV status. The purpose of this study was to understand adolescents' experience of ethical issues when attending HIV care and treatment that influenced their adherence to ART in Temeke regional referral hospital, Tanzania.

\section{Methods}

An exploratory, qualitative, descriptive design was used to examine adolescents' experience of ethical issues that influence their enrollment in and adherence to ART in Temeke Regional Hospital, Tanzania. This design was appropriate to first, gain knowledge of a poorly understood phenomenon concerning the ethical challenges that 
influence adolescents to enroll in and adhere to treatment within the African context; and second, describe the events and experiences of adolescents at Care and Treatment (CTC) (22). Adolescents participated in semistructured interviews, as described below.

\section{Data Collection}

This qualitative study was part of a larger quantitative study to assess knowledge of ethical issues among adolescents living with HIV and their parents and health care providers. Data were collected through semistructured interviews. The interview guide was developed from a literature review of ethical issues that influence adherence to ART among adolescents and in consultation with qualitative experts on the study team (24-27). Prior to full data collection, a small pilot study of 4 adolescents was conducted at Amana Regional Referral Hospital in Tanzania. This site was selected because it is similar to Temeke Regional Referral Hospital for care and treatment for HIV and AIDS in Dar es Salaam. Minor adjustments were made to several interview questions for clarity. Research assistants were trained for the purpose of data collection before going to the field.

Parents of adolescents with an appointment at the clinic were phoned by a "leader" of the adolescents to reaffirm their appointments. These leaders are adolescents selected to lead others in clinic activities, and their role is to assist in setting appointments for their peer group and to make follow-up appointments for those who did not show up for their appointment. The principal investigator (PI) worked with these leaders to phone adolescents who were on the list for the day to come with their parents to ask for their permission to participate in the study. On arrival, the PI introduced parents to the study and asked them if they would be interested in participating. A total of 28 were approached but six declined; five parents declined on behalf of their adolescent, and one adolescent personally declined. Parents who consented to participate were given a questionnaire for the quantitative aspect of the study. The PI purposely selected participants based on their age (10-19), education level (primary school, secondary school, Vocational Education and Training Authority students and university students), ability to express themselves, knowledge of their positive HIV status, and enrollment in ART. Selected adolescents participated in in-depth interviews. Further recruitment was stopped, because saturation of the data was reached after interviews with 22 adolescents.

Research assistants conducted in-depth interviews in private rooms. The interview guide and interviews were in Swahili-the national language comfortable to all participants. The average duration of each interview was 25 minutes. The interview guide provided open-ended questions to solicit participants' descriptions of their experiences of ethical issues during CTC attendance. Participants described how such issues influenced their enrollment in and adherence to ART. Probe questions were asked in areas that needed more clarification or elaboration. Interviews were audiotaped with participants' consent and transcribed verbatim; transcripts were translated to English. The study was conducted from January to August 2020.

\section{Data Analysis and Qualitative Rigor}

Descriptive statistics were used to analyze participants' demographic characteristics, including age, gender, duration of ART, age at enrollment in the CTC, and age when the individual learned of his/her HIV status. After transcription, each transcript was uploaded to NVivo 12 software, which assisted in data coding. Open coding was also conducted on printed hard copies before uploading them to the software. We used the principles of thematic analysis to explore adolescents' experience of ethical challenges in attending CTC and using ART (28). Thematic analysis helps researchers identify and examine themes or patterns within a particular context. 
Six steps for thematic analysis were followed (22). First, all transcripts were reviewed by the PI and coinvestigator to assess validity of data and become familiar with the data. Second, phrases, sentences, and paragraphs were organized in a meaningful way to form codes. We used open coding; codes were developed and modified as the coding process progressed. Third, we examined related codes, and codes were then refined into categories. Some of them were collated into initial themes. At the end of this step, the codes were organized into broader themes that seemed to specifically describe ethical issues in adolescents' HIV care and treatment. Fourth, themes were reviewed to identify whether they were supported by the data. Themes that were related or seemed to address the same issue or concern were combined to form a single theme, and those that were not supported by data were eliminated. Lastly, relationships among the themes were interpreted and described in connection to participants' responses.

Several criteria were used to evaluate the trustworthiness of the qualitative data. First, credibility was enhanced because the first author had spent time in the field and was familiar with the setting. Second, the various perspectives of the research team members, who had different degrees of familiarity with the setting, were included. Third, transferability was assessed, which is a determination of how applicable the findings are to other contexts and is also a way of evaluating trustworthiness of data (29). Here, the transcripts were translated to English, and discussion of emerging themes among research team members enriched the interpretation of the data through the balance of perspectives representing different backgrounds and qualitative expertise. These measures enhanced the credibility of the representation of participants' views presented here. Our detailed description of the study context, selection criteria, data collection, and analytical process is complemented below by quotations from participant interviews, which allow readers to judge the dependability of the analysis and transferability of the findings.

\section{Results}

\section{Participant Demographics}

In the sample of 22 adolescents interviewed, ages ranged from 13 to 19, with a mean of 16.6 years. Adolescents were generally evenly represented by gender, with 12 females and ten males. From an educational perspective, three were in primary school, ten were in secondary school, five were Vocational Education and Training Authority students, one was in adult education classes, and one was a university student. The remaining two participants had completed primary school, and both were employed. All participants knew their HIV status; 16 were tested at a young age (less than 5 years), and six were tested at age 12 years or older. Age at disclosure of HIV status to adolescents ranged from 10 to 18 years (mean $=11.5)$.

\section{Qualitative Themes}

Several themes emerged from the data: adolescents' lack of participation in decisions about HIV care and treatment; issues around disclosure of HIV status, such as delays in disclosure to the adolescent and disclosure to other persons and benefits and harms of such disclosures; and factors supporting and interfering with adherence to ART, such as parental support, organizational (clinic) support and problems, and selfstigmatization and shame.

Lack of autonomous decision making regarding enrollment in care and treatment of HIV. 
As noted, 16 participants were tested when they were younger than age 5 and did not have the capacity to decide to enroll in care and treatment for their HIV. Decisions about testing and enrollment were made by their parents, mostly mothers. Regardless of who made the decision about enrollment in care and treatment, adolescents reported adhering to their medication for their own health benefit, as signified by the quotes below:

"I was born with this disease; therefore, I was enrolled when still very young-my mother made the decision. I take medicine by adhering to treatment because I am HIV infected, therefore, I take ART in order to improve my body immunity." (Adolescent \# 09)

"I was enrolled when very young-my mother decided for me to be tested and enrolled in HIV care and treatment.. . I do take medicine in my mother's room." (Adolescent \#01)

Six adolescents were tested and enrolled in care and treatment for HIV at an older age (12-16 years). This decision was made by their parents or other relatives, such as an aunt or grandfather. These adolescents reported that they were not involved in the decision-making process and were unhappy about their lack of participation in matters involving them. Even though they were unhappy, they still took their medicine as noted below.

"I was tested at age 12, and I was very sick-hence not involved in the decision to test. After the HIV testing, disclosure was done to my grandfather, who later on informed me. I felt sad in my heart." (Adolescent \#04)

"I was tested when I was 15 years, I was not involved in the decision to test. I felt bad but I did not regard it as a mistake; for me it was a kind of love from my mother because through that I knew my health status that am living with HIV, this helped me to start medication early. I think if I did not start medication early, I would have already passed away." (Adolescent \# 03)

This participant also added that;

"Adolescents have to be given chance to participate in the decision making for medical care. I do not blame my mother for what she did, but adolescents have to exercise their autonomy in the matters that affect their life." (Adolescent \# 03)

\section{Disclosure of HIV status to the adolescent}

\section{Delays in disclosure leading to poor adherence to ART}

Most adolescents reported a delay in disclosure of their HIV status to themselves. Nondisclosure in some adolescents resulted in poor adherence to medication and deterioration of health status. Some of the adolescents felt that they were HIV infected even before they were informed, as reported in the following quotes.

"My father gave me this information about my health status when I was eleven years old; he told me that by now you are matured person, and you are living with HIV virus from your mother. I was heartbroken because I did not know what was going on, they were hiding this information." (Adolescent \# 09)

"I was told by my mother when I was twelve years after asking her why am I using medicine for a long time. Through attending clubs, I suspected I may be HIV infected. In clubs we were taught issues that made me know 
my health status." (Adolescent \# 22)

"My mother passed away, and I refused to take medicine until I knew why I was taking these medicines; at first I was told to take medicine because they accelerate growth since I was so thin. My health deteriorated after stopping ART." (Adolescent \# 11)

\section{Disclosure of adolescents' HIV status to others Benefit of disclosure of HIV status of adolescents to others}

Participants revealed that disclosing HIV infection to others, including friends, teachers, relatives, and matrons for adolescent students living in hostels, is important to support HIV-infected adolescents to take their medicine appropriately and without missing clinic appointments. Several quotes are provided below.

"I told my teachers myself, because I wanted them to know why I was not attending school sometimes; hospital administration wrote a letter and was submitted to school by my brother. Also, one of my friends at school knows my health status; she helps me when I got sick. I was in a critical health condition, so I decided to tell her. She didn't believe but she later on accepted." (Adolescent \# 21)

"My class teacher was aware about this situation, since he is the one who was responsible to give me permission to attend clinic.. .. My parents informed him about my situation." (Adolescent \# 04)

"Only my form two teacher knows my health status. It was a day whereby students who had problems, like the deaf, disabled, were explaining their problems, so the teacher said whoever knows that she/he is sick has to report, and being with HIV infection, I have to report." (Adolescent \# 01)

\section{Harm of disclosure of HIV status of adolescents to others}

Some adolescents reported being stigmatized and called names after HIV disclosure to others. One adolescent did not feel comfortable going to university for fear of other university students knowing her HIV status.

"My father is very supportive in my care. My mother died when I was young, and I am living with my father and stepmother, who insults me due to HIV infection and the need to go for medicine always. We do fight a lot, and she always lies about me. She as well discloses my HIV status to everyone who comes to our family, without caring for the impact of discrimination to me. I sometimes feel it is better if I was not born." (Adolescent \# 09)

"The challenge that we are facing is great-for example, I was selected to join a government University in Dodoma, which is good and cheaper, but I decided to find a private college in Dar es Salaam for fear of colleagues finding out about my HIV status when I have to move to hostel." (Adolescent \# 20).

\section{Adolescent's experiences that influenced the decision to adhere to ART}

\section{Parental support to their adolescents to adhere to ART}

Participants raised concerns about their inability to attend clinic despite understanding the importance of ART. They needed their parental support in logistical and financial concerns, such as the availability of bus fare, food, and drinks to come to the hospital. These affected their adherence, as indicated in the quotes below. 
"It is a personal decision to come to clinic to get medicine, counselling on reproductive issues and other services, but I do not want to come here due to cost of fare and food." (Adolescent \#21)

"It costs me because sometimes I can wake up without having bus fare, sometime when I attend here my friends are eating food, but I cannot afford to eat because I do not have money to buy food." (Adolescent \# 22)

"Bus fare, sometimes I do not manage to get bus fare, sometimes I do not get money for lunch, so I may stay the whole day at the clinic without eating. My parents are supportive in my care, since I was young and suffered HIV. Many parents might have already given up and foregone me, but my parents are taking care of me."(Adolescent \# 05)

"It is the bus fare-if it happens that those whom I am living with have no money, then I have to find other relatives who can help." (Adolescent \# 04)

\section{Health care organizational factors}

Participants reported that health care organizational factors are important to their adherence to ART, including time spent waiting for care, availability of medicine, health education provided at the clinic, adolescents' HIV clubs (organized groups of HIV-infected adolescents with the aim of encouraging one another), and attitudes of health care workers.

"I do not want to come to clinic, it is costly, I need time, there are traffic jams on the way to hospital, and I miss classes at school." (Adolescent \# 20)

"We delay at the hospital, there is the shortage of medicine; living far away this causes me to arrive at the hospital beyond the time for services and necessitates not getting medicines." (Adolescent \# 11)

"Health care workers are coming to work late, hence starting clinic late-therefore, delaying us unnecessarily." (Adolescent \# 22)

"The challenge that I face is time; sometime I take medicine late because the person who is distributing cards reports late to clinic." (Adolescent \# 15)

"Initially clinic was on Saturday, but this year I have to come on Friday; it is difficult to attend clinic on Friday because I have classes at school." (Adolescent \# 01)

"Sometimes some doctors have bad statements towards patients; we stay for a long time waiting for services while doctors are chatting or eating without bearing in mind that patients have other societal obligations." (Adolescent \#07)

\section{Individual adolescent factors}

Some adolescents reported self-stigmatization and blaming themselves for being HIV infected. This affects their decision to adhere to care and treatment as shown by the quotes below:

"I do hide; I hide myself a lot when taking ART because I do not want anyone to know that I am HIV infected." (Adolescent \# 01) 
On further inquiry he reported, "I do not want people to stigmatize my mother; she is petty trader, and if they cannot buy from her, we can suffer." (Adolescent \# 01)

"I do not want to attend clinic due to feeling of shame when going to HIV care and treatment clinic to take medicine." (Adolescent 15)

\section{Discussion}

This is one of the first studies to focus on adolescents' experience of participating in a care and treatment clinic for HIV in Tanzania. Several ethical concerns were noted. First, adolescents sought autonomy and some control in decision making about their health and well-being, but many found this difficult to do because such decisions were made before they became aware of their HIV status. Second, some adolescents felt shame, stigma, and worthlessness associated with their HIV status. Third, delays in disclosure of HIV status to adolescents resulted in some adolescents neglecting ART and a worsening of their health status. Disclosure to others was regarded as both beneficial and harmful.

Individual autonomy is an important ethical requirement for everyone, including adolescents in low-to middleincome countries (24). The results of this study, however, show a lack of adolescents' involvement in decisionmaking about HIV testing as well as enrollment in ART. Guidelines to involve adolescents in their testing decision vary from one practice setting to another (30). In South Africa, adolescents from age 12 onward can consent for HIV testing (31), while in Tanzania the age for consent for HIV testing is 16 (32). Most adolescents in our study were tested at a young age and were not able to deliberate about testing for HIV and its implications. Parents make decisions in their children's best interest, and findings from this study reveal that adolescents appreciated their parents love for them. However, our participants also wanted to make decisions for themselves. Despite being HIV tested and enrolled in care in the CTC without their consent, adolescents adhered to ART because they believed it improved their health. Hosek and colleagues conducted a qualitative inquiry and found similar results with U.S. adolescents, who adhered to their medication because they wanted to stay healthy and alive (33). Six adolescents in our study were tested between the ages of 12 and 16; however, they were not involved in decision to test and enroll them in care. These adolescents felt that they had been ignored, but they adhered to medication for their own health benefit.

Despite their perception of a lack of autonomy, all adolescents who participated in this study adhered to HIV care and treatment and attended the CTC. Parental support was a key factor in reminding adolescents to attend the clinic; emotional and economic support were also among the issues mentioned by adolescents as a factor in adherence to ART. This finding is similar to findings of a study conducted in Brazil (34). In that study, adolescents living in a stable family structure had better adherence to medication and were less likely to face ethical challenges like stigma and discrimination. This finding is also supported by other studies (35).

Participants also reported feelings of personal shame that they are living with HIV, which resulted in neglecting appointments and in not taking their ART on time. Self-stigmatization and feelings of worthlessness were among the individual factors reported by our study participants as reasons for poor adherence to medication. A similar study conducted with adults in Northern Tanzania reported that stigma and involuntary disclosure (i.e., learning about one's HIV status accidentally) were associated with poor adherence to ART (36). Another factor mentioned by adolescents was tight school schedules, which led to forgetting their medicine or even ignoring 
ART when they were hungry and busy. ART side effects, such nausea, dizziness, and drowsiness, can affect adolescents in their day-to-day activities; however, adolescents' life depends on consistent adherence to ART. The adolescents in our study knew that they had no choice to decline medication if they wanted to survive.

Disclosure of HIV status is an important step in adolescents' adherence to medication and their growth and development (37). Unfortunately, disclosure of HIV status to adolescents is delayed in many situations (38). The current study found that adolescents were left wondering why they were taking medicine daily. For some study participants, nondisclosure resulted in poor adherence to medication and deterioration of their health status. Other studies have shown the same result $(17,39)$.

Adolescents' HIV clubs have been found to improve adherence to medication, especially for those ages 15 to 19 (40). In adolescent clubs, they are taught about secondary HIV infection and prevention of infection by being responsible and not infecting their partner (41). Some adolescents in our study reported attending such clubs before their HIV status was disclosed to them-that is, some "got the hint" that they were HIV positive before being told. Uninformed adolescents may wonder why no one is telling them. Knowledge of one's HIV status is challenging to adolescents, and they may become sad and experience self-stigma, as our data show. But after a period of time, they may learn to live with HIV and assume the responsibilities associated with their disease.

Some of our adolescent participants informed their teachers about their HIV status. Such disclosure helped adolescents keep clinic appointments without worries or fears of missing school. Friends also supported sick adolescents when they knew they had HIV, which also improved their adherence to their medication. In some instances, adolescents informed their siblings, who also conferred emotional support whenever they needed it (42).

Disclosure is an ethical dilemma, because despite its benefits it may also lead to stigma and discrimination, which are physically and emotionally harmful for HIV-infected adolescents. Some participants were ostracized by their relatives because of their HIV status. Some participants also reported being bullied and segregated from others. Disclosure to their sexual partners led to broken relationships and fears of not being able to become engaged again. HIV-infected individuals have a duty to warn sexual partners and to give their partner the chance to make an informed choice. But this may result in stigma and discrimination against the HIV-infected individual (43).

The health care facility environment and adolescent friendliness are among the factors that facilitated adolescents' decision to attend the HIV CTC. This finding is also supported by other studies (44). Disrespectful providers discouraged CTC attendance. This finding is similar to a finding in a 2014 study in which participants reported clinic friendliness as important to adolescents (45). This included flexibility in the days and times of the clinic opening that did not collide with school activities. In that study, service providers with good language skills and infrastructure that protected adolescents' privacy and confidentiality in terms of their HIV status were also important. In the current study, the time that adolescents spent waiting for their service and providers' attitudes were also among the main motivators for adolescents to come to the hospital clinic.

Finally, participants also mentioned adolescents' HIV clubs as one of the health care organizational factors that helped them adhere to ART. HIV clubs provide services that include reproductive health services, ART adherence counselling, and an entrepreneurship course. This type of support system can help adolescents living with HIV combat their life challenges with hope and enthusiasm. Other studies have shown teen clubs to be helpful in 
adolescent retention in HIV care and ART (41). More research on the benefits and challenges of this type of adolescent support is needed.

\section{Limitations}

There were several limitations associated with this study. First, the study interviewed individuals who agreed to participate. This may have created a selection bias, and those who chose not to participate may have different concerns not readily addressed in this study. These adolescents may have been uncomfortable discussing their views on parental authority versus adolescent autonomy in decision making for HIV services, on HIV disclosure to others and sexual partners, and on health care workers and the hospital environment in general. To address this limitation, however, the adolescents were told that the researcher would keep their information confidential. In addition, the interviews were conducted in a private room where adolescents were comfortable and away from any outside influences. Generalization of findings from this study is limited to the population of interest, because it was conducted with adolescents at one hospital clinical in Tanzania. Future research should examine these issues at other types of clinics and with other adolescents, parents, and providers across different regions of the country. Finally, the smaller sample size limits generalizability. However, the qualitative nature of the study provides significant insights, and the greatest strength of a qualitative study is the depth and richness of exploration and description it offers (46).

\section{Conclusion}

Disclosure of HIV status to the adolescent is an ethical dilemma in HIV care of adolescents, but it also can have a positive influence on the adolescent's life in terms of adherence to ART. Knowledge and adherence can allow adolescents to live a life of purpose and to have hope for the future. Further research is needed on how to involve adolescents in their HIV care and to identify what is important to them as they learn to live with a chronic illness. Their voices are critical in developing strategies and guidelines to improve care delivery for this population. More research is also needed on the role of parental support, compared with adolescents' HIV clubs, in helping adolescents understand their disease, their disease trajectory, and their responsibilities as they age. Adolescents are the future; we must continue to respect their views and understand their health needs as they live with a chronic condition that can bring many challenges to their life and the relationships that they develop.

\section{Abbreviations}

AIDS Acquired Immunodeficiency Syndrome

ART Antiretroviral Therapy

CTC Care and Treatment

NatREC National Research Ethics Committee

MUHAS Muhimbili University of Health and Allied Sciences

UNICEF United Nations Children's Fund 


\section{Declarations}

\section{Ethical Consideration}

This research was carried out in accordance to national and international guidelines of human subject protection in research including; National Institute of Medical Research Standard Operating Procedure for research, Council of International Organization for Medical Science CIOMS and Declarations of Helsinki. Ethical clearance was obtained from Muhimbili University of Health and Allied Sciences (MUHAS) Institutional Review Board and the National Research Ethics Review Committee (NatREC). Permission to collect data was also secured from Temeke Regional Referral Hospital's authority. Written informed consent and pediatric assent was obtained from parents with adolescents aged 10 to 17 years (23). Older adolescents (18- and 19-year-old) consented on their own.

\section{Consent for publication}

Participants' photos and audios or any identifiable information are not included in this publication.

\section{Availability of data and material}

Data was entered into NVIVO software for analysis, audios and transcripts are available in computer not uploaded online. Data will be available upon request from corresponding author.

\section{Competing interest}

None

\section{Funding:}

This research was sponsored by Dartmouth MUHAS Research Ethics Training Program (DMRET), Grants number: 5R25TW007693 offered as small grant for independent research which was used to compensate research participants and research assistants for their field work and Ministry of Education, Science and Technology which sponsored this PhD studies without which I would not have the opportunity to conduct the research: FB.84/370/02A/83

\section{Authors contributions:}

RSJ, Proposal development, seeking ethical clearance, data collection, data analysis, manuscript preparation and submission for publication

GRM, Editing Proposal, Data analysis, Manuscript reading and editing

GF, Main supervisor; Proposal development, data analysis, Manuscript preparation

$\mathrm{CMU}$, Co supervisor; Proposal development, data analysis, Manuscript preparation

All authors have read and accepted manuscript to be submitted.

\section{Acknowledgement:}


Temeke Regional Referral Hospital CTC staffs, Tatu the peer leader who communicated and arranged our meeting with parents for consent to participate in the study, research participants both parents and adolescents for their time, patience and information and research assistants, Mr. Emmanuel Emmachius and Vedasto Osward for assisting in participant's enrollment and data collection.

\section{References}

1. Armstrong A, Caswell G, Kihara, Cecilia Kate L. Good Practice Guide: Adolescent HIV programming READYHere we come! 2017;60. Available from:

2. Ford CA, English A. Limiting confidentiality of adolescent health services: what are the risks? JAMA. 2002;288(6):752-3.

3. Grootens-Wiegers P, Hein IM, van den Broek JM, de Vries MC. Medical decision-making in children and adolescents: Developmental and neuroscientific aspects. BMC Pediatr. 2017;17(1):1-10.

4. Dickey SB, Deatrick J. Autonomy and decision making for health promotion in adolescence.pdf. Pediatr Nurs. 2000;26(5):481-2.

5. Korsch BM, Gozzi EK, Francis V. Gaps in doctor-patient communication: I. Doctor-patient interaction and patient satisfaction. Pediatrics. 1968;42(5):855-71.

6. Unicef-Tanzania. Tanzania adolescent report. 2011.

7. National Bureau of Statistics (NBS). The United Republic of Tanzania 2015 TANZANIA IN FIGURES. 2016; Available from: http://www.nbs.go.tz/nbs/takwimu/references/Tanzania_in_Figures_2015.pdf

8. AVERT. HIV and AIDS in Tanzania Key affected populations in Tanzania Women and HIV in Tanzania. 2013;1-6.

9. Tanzania Bureau of Statistics. Tanzania Demographic and Health Survey 2016. Natl Bur Stat Dar es Salaam, Tanzania ICF Macro Calverton, Maryland, USA [Internet]. 2016;1-482. Available from: http://www.measuredhs.com/pubs/pdf/FR243/FR243\%5B24June2011\%5D.pdf

10. Busza J, Strode A, Dauya E, Ferrand RA. Falling through the gaps: How should HIV programmes respond to families that persistently deny treatment to children? Vol. 19, Journal of the International AIDS Society. 2016. p. 11-3.

11. UNAIDS. Seen but not heard: Very young adolescents aged $10-14$ years. 2004;

12. UNICEF. AIDS HIV and AIDS. 2020;1-6. Available from: https://www.unicef.org/tanzania/media/2436/file/HIV Programme Fact Sheet.pdf

13. Wiener L, Mellins CA, Marhefka S, Battles HB. Disclosure of an HIV diagnosis to children: history, current research, and future directions. J Dev Behav Pediatr [Internet]. 2007;28(2):155-66. Available from: http://www.ncbi.nlm.nih.gov/pubmed/17435473\%5Cnhttp://www.pubmedcentral.nih.gov/articlerender.fcgi? artid=PMC2440688

14. Knapp van Bogaert D, Ogunbanjo GA. Common ethical issues related to HIV/AIDS. South African Fam Pract. 2011;53(6):605-9.

15. Veinot TC, Flicker SE, Skinner HA, McClelland A, Saulnier P, Read SE, et al. "Supposed to make you better but it doesn't really": HIV-positive youths' perceptions of HIV treatment. J Adolesc Heal. 2006;38(3):261-7. 
16. Becker MH, Mainman LA. Sociobehavioral Determinants of Compliance with Health and Medical Care Recommendations. Med Care [Internet]. 1975;13(1):10-24. Available from:

http://www.udgvirtual.udg.mx/paakat/index.php/paakat/rt/printerFriendly/519/661

17. Mengesha MM, Ajema D, Teshome A, Tura AK. The association between diagnosis disclosure and adherence to antiretroviral therapy among adolescents living with HIV in sub-Saharan Africa: A protocol for systematic review and meta-analysis. Syst Rev. 2020;9(1):1-5.

18. http://www.sciencedirect.com/science/article/pii/S0002937807002967

19. Kiula CS, Majapa S, Mwansisya TE. Disclosure of HIV status of school-age children: an experience from health-care providers and children's caregivers in Dar es Salaam, Tanzania. Vulnerable Child Youth Stud. 2019 Jul 3;14(3):270-3.

20. Southern African HIV Clinicians S. Young people, HIV and AIDS The medical advances that have transformed HIV treatment have yet to alter the stark reality for young people, particularly in low to. Guidel adherence to Antiretrovir Ther Adolesc young adult. 2017;1-21.

21. Sanga Z, Kapanda G, Msuya S, Mwangi R. Factors influencing the uptake of Voluntary HIV Counseling and Testing among secondary school students in Arusha City, Tanzania: a cross sectional study. BMC Public Health [Internet]. 2015;15(1):452. Available from:

http://bmcpublichealth.biomedcentral.com/articles/10.1186/s12889-015-1771-9

22. Kim H, Sefcik JS, Bradway C. Characteristics of Qualitative Descriptive Studies: A Systematic Review. Res Nurs Heal [Internet]. 2017;40(1):23-42. Available from: http://login.ezproxy.lib.umn.edu/login? url=http://search.ebscohost.com/login.aspx? direct=true\&AuthType=ip,uid\&db=eric\&AN=EJ1069509\&site=ehost-live

23. WHO. Handout for Module A Introduction. 2018. 6 p.

24. Beauchamp TL, Childress J. Principles of Biomedical Ethics, New York Press. New York; 2001. 11 p.

25. Heubel F, Biller-andorno N. The contribution of Kantian moral theory to contemporary medical ethics: A critical analysis. 2005;5-18.

26. Kant I. Groundwork for the Metaphysic of Morals. 2017;

27. Misselbrook D. The ReviewAn A-Z of medical philosophy Duty, Kant, and Deontology. Br J Gen Pract. 2013;63(609):211.

28. Braun V, Clarke V. Using thematic analysis in psychology. Qual Res Psychol. 2006;3(2):77-101.

29. Lincoln Y, Guba E. Naturalistic inquiry. Brevely hill, editor. 55 City Road London EC1Y 1SP: SAGE Publications; 1985.

30. Fox K, Ferguson J, Ajose W, Singh J, Marum E, Baggaley R. Adolescent consent to testing: a review of current policies and issues in sub-Saharan Africa. HIV Adolesc Guid HIV Test Couns care Adolesc living with HIV Recomm a public Heal approach considerations policy-makers Manag. 2013;1-4.

31. Sam-Agudu NA, Folayan MO, Ezeanolue EE. Seeking wider access to HIV testing for adolescents in subSaharan Africa. Pediatr Res. 2016;79(6):838-45.

32. National AIDS Control Program.

Tanzania_national_guideline_for_management_hiv_and_aids_may_2015._tagged. 2015;

33. Hosek SG, Harper GW, Domanico R. Psychological and social difficulties of adolescents living with hiv: A qualitative analysis. J Sex Educ Ther. 2000;25(4):269-76.

Page 14/15 
34. Galano E, Turato ER, Delmas P, Côté J, De Fátima Thomé Barbosa Gouvea A, De Menezes Succi RC, et al. Experiences of adolescents seropositive for HIV/AIDS: A qualitative study. Rev Paul Pediatr [Internet]. 2016;34(2):171-7. Available from: http://dx.doi.org/10.1016/j.rppede.2015.08.019

35. Nabukeera-Barungi N, Elyanu P, Asire B, Katureebe C, Lukabwe I, Namusoke E, et al. Adherence to antiretroviral therapy and retention in care for adolescents living with HIV from 10 districts in Uganda. BMC Infect Dis. 2015;15(1).

36. Lyimo RA, Stutterheim SE, Hospers HJ, De Glee T, van der Ven A, De Bruin M. Stigma, disclosure, coping, and medication adherence among people living with HIV/AIDS in northern Tanzania. AIDS Patient Care STDS [Internet]. 2014;28(2):98-105. Available from: http://www.ncbi.nlm.nih.gov/pubmed/24517541

37. Arrivé E, Dicko F, Amghar H, Aka AE, Dior H, Bouah B, et al. HIV status disclosure and retention in care in HIVinfected adolescents on antiretroviral therapy (ART) in West Africa. PLoS One. 2012;7(3):1-8.

38. Montalto GJ, Sawe FK, Miruka A, Maswai J, Kiptoo I, Aoko A, et al. Diagnosis disclosure to adolescents living with HIV in rural Kenya improves antiretroviral therapy adherence and immunologic outcomes: A retrospective cohort study. PLoS One. 2017;12(10):1-11.

39. Madiba S, Diko C. The Consequences of Delaying Telling Children with Perinatal HIV About Their Diagnosis as Perceived by Healthcare Workers in the Eastern Cape; A Qualitative Study. Children. 2020;7(12):289.

40. MacKenzie RK, van Lettow M, Gondwe C, Nyirongo J, Singano V, Banda V, et al. Greater retention in care among adolescents on antiretroviral treatment accessing "Teen Club" an adolescent-centred differentiated care model compared with standard of care: a nested case-control study at a tertiary referral hospital in Malawi. J Int AIDS Soc. 2017;20(3).

41. Munyayi FK, van Wyk B. The effects of teen clubs on retention in HIV care among adolescents in Windhoek, Namibia. South Afr J HIV Med. 2020;21(1):1-9.

42. Kimera E, Vindevogel S, Rubaihayo J, Reynaert D, De Maeyer J, Engelen AM, et al. Youth living with HIV/AIDS in secondary schools: perspectives of peer educators and patron teachers in Western Uganda on stressors and supports. Sahara J. 2019;16(1):51-61.

43. Mburu G, Hodgson I, Kalibala S, Haamujompa C, Cataldo F, Lowenthal ED, et al. Adolescent HIV disclosure in Zambia: Barriers, facilitators and outcomes. J Int AIDS Soc. 2014;17.

44. Lamb MR, Fayorsey R, Nuwagaba-Biribonwoha H, Viola V, Mutabazi V, Alwar T, et al. High attrition before and after ART initiation among youth (15-24 years of age) enrolled in HIV care. Aids. 2014;28(4):559-68.

45. Layer EH, Kennedy CE, Beckham SW, Mbwambo JK, Likindikoki S, Davis WW, et al. Multi-Level Factors Affecting Entry into and Engagement in the HIV Continuum of Care in Iringa, Tanzania. 2014;9(8).

46. Myers M. Qualitative Research and the Generalizability Question: Standing Firm with Proteus by Margaret Myers, RN, BA, BscN, EdD (Cand). Vol. 4. 2000. 2000 p. 